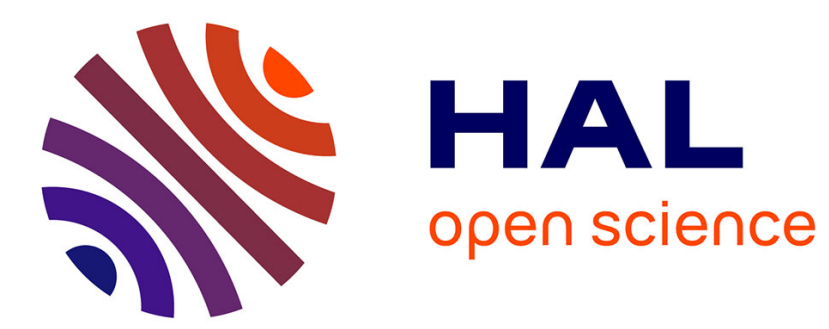

\title{
LSE method using the CHSS model
}

\author{
Pascal Lantieri, Francis Guerin, Sorin Voiculescu
}

\section{To cite this version:}

Pascal Lantieri, Francis Guerin, Sorin Voiculescu. LSE method using the CHSS model. Journal of Statistical Planning and Inference, 2009, 139 (5), pp.1809-1820. 10.1016/j.jspi.2008.05.031 . hal00881225

\section{HAL Id: hal-00881225 \\ https://hal.science/hal-00881225}

Submitted on 7 Nov 2013

HAL is a multi-disciplinary open access archive for the deposit and dissemination of scientific research documents, whether they are published or not. The documents may come from teaching and research institutions in France or abroad, or from public or private research centers.
L'archive ouverte pluridisciplinaire HAL, est destinée au dépôt et à la diffusion de documents scientifiques de niveau recherche, publiés ou non, émanant des établissements d'enseignement et de recherche français ou étrangers, des laboratoires publics ou privés. 


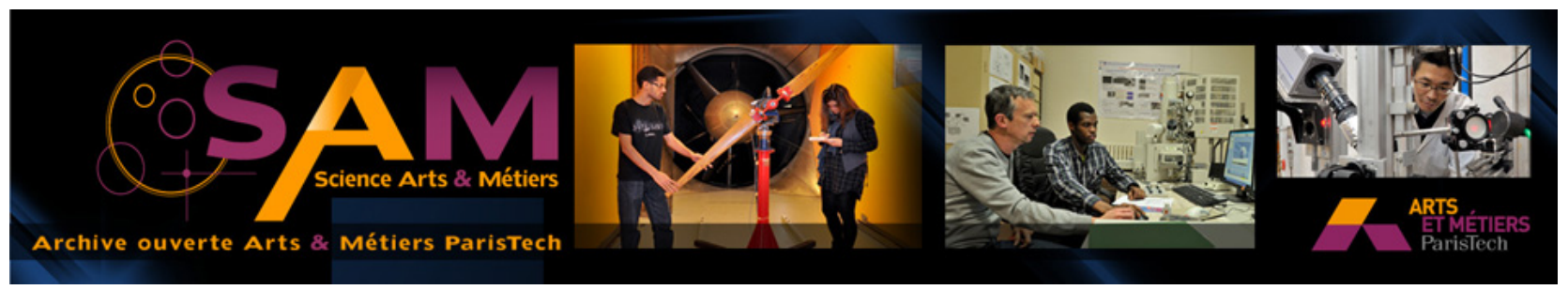

Science Arts \& Métiers (SAM)

is an open access repository that collects the work of Arts et Métiers ParisTech researchers and makes it freely available over the web where possible.

This is an author-deposited version published in: http://sam.ensam.eu Handle ID: .http://hdl.handle.net/10985/7479

\section{To cite this version :}

Pascal LANTIERI, Francis GUERIN, Sorin VOICULESCU - LSE method using the CHSS model Journal of Statistical Planning and Inference - Vol. 139, n5, p.1809-1820 - 2009 


\title{
LSE method using the CHSS model
}

\author{
P. Lantiéria,b,*, F. Guérin ${ }^{\mathrm{b}}$, S. Voiculescu ${ }^{\mathrm{b}}$ \\ ${ }^{a}$ Ecole Nationale Supérieure d'Arts et Métiers, 2 Bd du Ronceray, 49100 Angers, France \\ ${ }^{\mathrm{b}}$ Quality and Reliability Department, University of Angers, France
}

Keywords:

Accelerated life testing

Step-stress test

Weibull distribution

Changing shape and scale

Least square estimation

Bootstrap

\begin{abstract}
A B S T R A C T
Testing the reliability at a nominal stress level may lead to extensive test time. Estimations of reliability parameters can be obtained faster thanks to step-stress accelerated life tests (ALT). Usually, a transfer functional defined among a given class of parametric functions is required, but Bagdonavičius and Nikulin showed that ALT tests are still possible without any assumption about this functional. When shape and scale parameters of the lifetime distribution change with the stress level, they suggested an ALT method using a model called CHanging Shape and Scale (CHSS). They estimated the lifetime parameters at the nominal stress with maximum likelihood estimation (MLE). However, this method usually requires an initialization of lifetime parameters, which may be difficult when no similar product has been tested before. This paper aims to face this issue by using an iterating least square estimation (LSE) method. It will enable one to initialize the optimization required to carry out the MLE and it will give estimations that can sometimes be better than those given by MLE.
\end{abstract}

\section{Introduction}

To define the lifetime distribution of highly reliable components, accelerated life testing (ALT) methods are often used. ALT implies that units are tested under higher stresses than nominal, so that failures occur sooner. Several models, called acceleration models, are often used to predict the lifetimes at the nominal stress from lifetimes at a higher one (see, for example, Nelson, 1990; Pham, 2003). The functional transforming lifetimes at a given stress into lifetimes at another one is called the time transfer function (TTF) (Bagdonavicius and Nikulin, 2001). When it is known, the survival function at the nominal stress can be estimated from ALT results. However, when very little information is known about the product, it is difficult to define such a model.

If the TTF is not exactly known, but can be chosen among a given class of parametric functions, it is possible to estimate all the parameters from the ALT results. This can be accomplished with different samples subjected separately to different stress levels (see Nelson, 1990) or with one single sample subjected to a step-stress accelerated test (see Mc Linn, 1998). This means that each unit is subjected to different stress levels as long as it works. Then, a failure times analysis can be processed using parametric estimation methods like maximum likelihood estimation (MLE) or least square estimation (LSE), as described by Tebbi et al. (2005).

When no class of parametric functions can be found for the TTF or for the lifetime distribution, it is still possible to predict the survival function at the nominal stress from ALT results. The only requirement is to define one of the steps at this nominal level. A convenient way to do this has been defined by Bagdonavicius and Nikulin (2001). They suggested a two part experiment. To begin with, the half of the sample is subjected to an uncensored test at a stress level $x_{1}$ higher than the nominal one $\left(x_{2}\right)$, and then, the second half of the sample is subjected to a two-step test at the stress levels $x_{1}$ and $x_{2}$.

\footnotetext{
* Corresponding author at: Arts et Metiers ParisTech, LPMI, 2 Bd du Ronceray 49100 Angers, France. Tel.: +33241 207381; fax: +33241226521.

E-mail address: pascal.lantieri@angers.ensam.fr (P. Lantiéri).
} 
For this plan, the survival function at stress $x_{2}$ can be defined either by a parametric method (if the class of lifetime distribution is known) or by a non-parametric method.

The models defined lead to transform failure times $t_{i}$ into equivalent failure times $t_{\mathrm{e} i}$, so that the survival function $S$ at $t_{i}$ defined for the two-step test equals the survival function $S_{2}$ at $t_{\mathrm{e} i}$ defined for a single step at the nominal stress $x_{2}$.

The first model defined by this way is called the "accelerated failure time" (AFT) model (Bagdonavicius and Nikulin, 1995). As it was not applicable to changing shape parameters, a second model, called "CHanging Shape and Scale" (CHSS) was defined. These two models both use an MLE method to estimate the lifetime distribution at the nominal stress level $x_{2}$. Although it has not been detailed yet for more than two steps, their method is also applicable for any step-stress test beginning with steps at increasing level and ending with a step at the nominal level.

As an example, to test a whole sample (in parallel) at the highest possible stress level without changing the failure mode, it may be useful to increase the level step by step with little increments. It enables one to record a significant number of failure times until the failure mode changes for one of the specimens. At this time, if the survival specimens are supposed undamaged by this new failure mode, they can be subjected to a lower level and their times to failure can be recorded too.

Thus, a lot of steps may be required to reach the highest possible stress level. Then, Nikulin and Bagdonavičius's CHSS method would lead to optimize a likelihood function with a lot of parameters. When their starting values are far from the true ones, it may not be consistent with current calculating capabilities.

To face this problem, we suggest estimating these parameters by a LSE method processing the failures step by step. For each step, the equivalent times can be fitted by a given probability plot to obtain estimations of specific parameters. After each estimation, this plot is modified by taking into account the equivalent failure times of the steps already processed. The plot obtained when all the failures are processed enables one to estimate the specific parameters at the nominal stress level.

As no likelihood function is used, the confidence intervals $(\mathrm{CI})$ found with the usual Fisher information matrix cannot be defined. However, they can be found by using a bootstrap method. The accuracy of parameter estimations and $\mathrm{Cl}$ found by this way will be verified on several simulations with different test profiles.

\section{Description of the AFT and CHSS methods}

Both models assume a continuous survival function $S$ and that the evolution of $S$ only depends on the stress level. It is the usual Sediakyn's principle (see Sedyakin, 1966), applicable for the most common damaging mechanisms.

This principle implies that the distribution of failure times $t_{i}$ during a step-stress test can be changed into a distribution of equivalent times $t_{\mathrm{ei}}$ at a single stress for which the survival functions will take the same values.

Their calculation method of equivalent times is shown for a decreasing step-stress test in Fig. 1.

The failure time distribution at the first step is usually expanded to obtain the equivalent times at the second stress level. The equivalent time $t_{\mathrm{e} i}$ of any failure time $t_{i}$ at the second step is obtained with the shift shown in Fig. 2 . The other steps are processed in the same way.

For a two-step stress with a second step at the nominal level, the AFT model leads to

$$
t_{\mathrm{e} i}=\left\{\begin{array}{l}
S_{2}^{-1} \circ S_{1}\left(t_{i}\right)=r \times t_{i} \text { for the first step }\left(t_{i} \leqslant \tau_{1}\right) \\
S_{2}^{-1} \circ S\left(t_{i}\right)=r \times \tau_{1}+t_{i}-\tau_{1} \text { for the second step }\left(t_{i}>\tau_{1}\right)
\end{array}\right.
$$

where $r$ is an unknown factor, $S_{k}(k=1$ or 2$)$ is the survival function defined for a test at the single stress level $x_{k}$, and $S$ is the survival function defined for the two-step test.

As this model is inaccurate for Weibull or normal distributions if shape parameters change with the stress level, a new model called CHSS model was defined (see Bagdonavicius et al., 2004). For a two-step test with the second step at the nominal level,

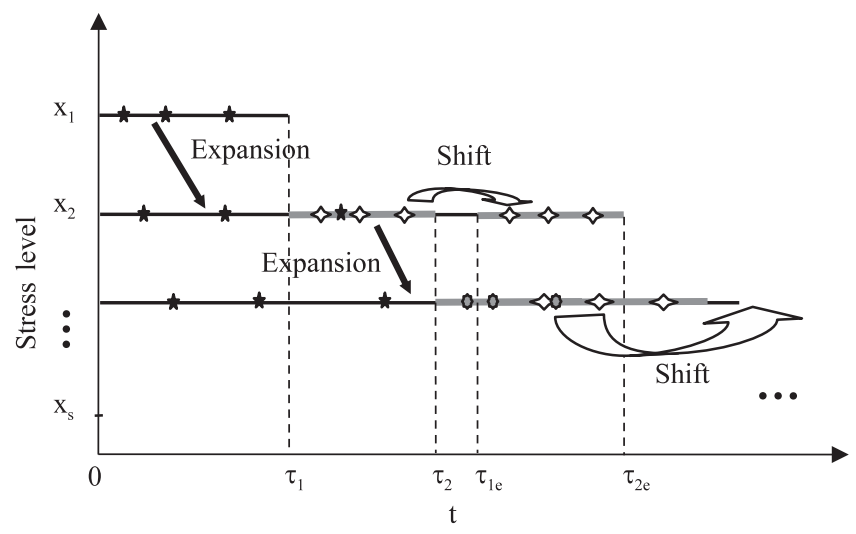

Fig. 1. Definition of equivalent time distributions. 


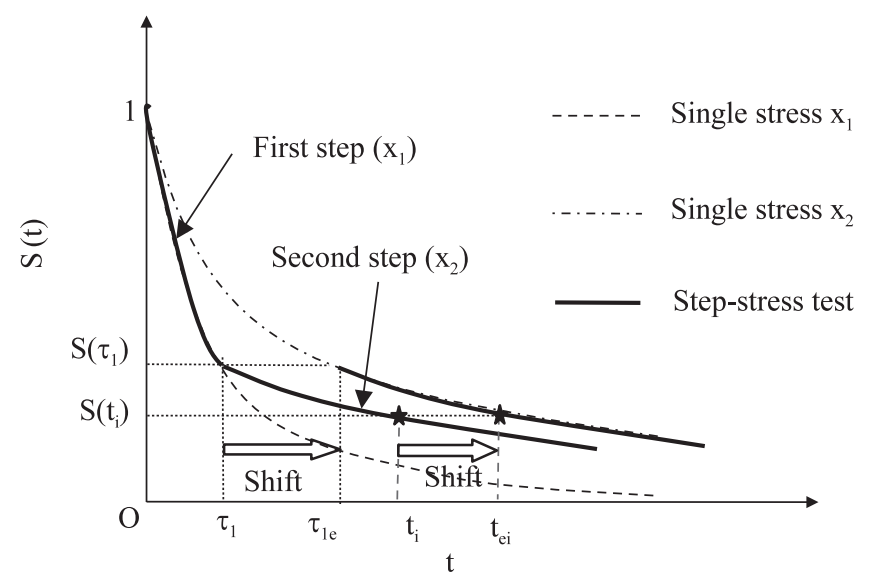

Fig. 2. Survival functions.

the CHSS model leads to

$$
t_{\mathrm{e} i}= \begin{cases}\eta_{2}\left(\frac{t_{i}}{\eta_{1}}\right)^{\beta_{1} / \beta_{2}} & \text { for the first step } \\ \eta_{2}\left(\frac{\tau_{1}}{\eta_{1}}\right)^{\beta_{1} / \beta_{2}}+t_{i}-\tau_{1} & \text { for the second step }\end{cases}
$$

where $\eta_{k}$ and $\beta_{k}$ are the unknown scale and shape parameters at the stress level $x_{k}$.

In both methods, equivalent times of the second step are calculated by shifting the true ones and specific parameters are estimated with an MLE method.

For $N_{1}$ failures at the first step and $N_{2}$ failures at the second step, the likelihood function is given by

$$
L\left(\eta_{1}, \beta_{1}, \eta_{2}, \beta_{2}\right)=\prod_{i=1}^{N_{1}} f_{1}\left(t_{i}\right) \times \prod_{i=N_{1}+1}^{N_{1}+N_{2}} f_{2}\left(t_{i}+A_{2}\right) \cdot S_{2}\left(\tau_{2}+A_{2}\right)^{N-N_{1}-N_{2}}
$$

where $A_{2}=\eta_{2}\left(\tau_{1} / \eta_{1}\right)^{\beta_{1} / \beta_{2}}-\tau_{1}$, and where $f_{k}$ and $S_{k}$ are the probability density functions and survival functions at the stress levels $x_{k}$ (with $k=1$ or 2 ).

Then, $\mathrm{CI}$ of estimations can be obtained by estimation of the Fisher information matrix by evaluating the second derivatives of the likelihood function $L$ at the ML estimates (see Nelson, 1990).

This method can also be carried out for any number of steps $s>2$. The likelihood function is defined for any lists of parameters $\eta=\left(\eta_{1}, \ldots, \eta_{s}\right)$ and $\beta=\left(\beta_{1}, \ldots, \beta_{s}\right)$ by

$$
L(\eta, \beta)=\prod_{k=1}^{s} \prod_{i=1}^{N_{k}} f_{k}\left(t_{i}^{(k)}+A_{k}\right) \times S_{s}\left(\tau_{s}+A_{s}\right)^{N-\sum_{k=1}^{s} N_{k}}
$$

where $t_{i}^{(k)}$ are the failure times at step $k(k \in[1, s]), A_{1}=0$ and $A_{k}=\eta_{k}\left(\left(\tau_{k-1}+A_{k-1}\right) / \eta_{k-1}\right)^{\beta_{k-1} / \beta_{k}}-\tau_{k-1}$ for any $k \in[2, s], f_{k}$ and $S_{k}$ are the probability density functions and survival functions at the stress levels $x_{k}(k \in[1, s])$.

This relationship means that any failure time $t_{i}^{(k)}$ at any step $k$ can be changed into an equivalent time $t_{\mathrm{e} i}^{(k)}$ corresponding to a test at a single stress level $x_{k}$. The previous relationships show that $t_{\mathrm{e} i}^{(k)}$ is simply shifted from $t_{i}^{(k)}$ as illustrated in Figs. 1 and 2. These relationships also show that, for any $k \geqslant 2$, the shift $A_{k}$ only depends on the parameters $\eta_{j}$ and $\beta_{j}, j=1-k$.

Moreover, for any number of steps, $\mathrm{Cl}$ can be obtained for all the parameters by using estimates of standard deviations obtained for the local estimate of the information Fisher matrix.

In practice, ML estimations can lead to problems. First of all, to converge to its maximum, the likelihood function may have to be initialized near the true values of parameters.

To avoid some of these problems, an iterative LSE processing failure times step by step will now be presented. It will be shown that the single parameter to initialize (for each step processing) can be found very close to its true value. Moreover, even if the likelihood function is not used, $\mathrm{CI}$ can still be obtained by using a bootstrap method.

The asymptotic behaviors of the two methods have not been compared yet but it is a work-in-progress. 


\section{Description of the iterative LSE method}

In this paper, the method will be described for Weibull distributions only but, with very few modifications, it can also be applied for normal or lognormal distributions. To be applicable to any step-stress profile, defined by any number of steps, the following notations will have to be used:

- $N$ the sample size,

- $N_{\mathrm{f}}$ the number of uncensored failures,

- $s$ the number of steps,

- $x_{k}(k \in[1, s])$, the stress level at step $k$,

- $\tau_{k}$ the time to the end of the step $k$,

- $t_{i}, i \in\left[1, N_{\mathrm{f}}\right]$, the uncensored failure times,

- $S$ the survival function defined for the step-stress test,

- $S_{k}$ the survival function defined for a test at a single stress level $x_{k}$,

- $\tau_{k, j}(k \in[0, s], j \in[0, s])$, the equivalent time defined by $\tau_{k, j}=S_{j}^{-1} \circ S\left(\tau_{k}\right)$,

- $t_{i, j}\left(i \in\left[0, N_{\mathrm{f}}\right], j \in[0, s]\right)$, the equivalent failure time defined by $t_{i, j}=S_{j}^{-1} \circ S\left(t_{i}\right)$,

- $\eta_{k}$ and $\beta_{k}$, the scale and shape parameters of the Weibull lifetime distribution at the single stress level $x_{k}$.

With these notations, the estimations of Weibull parameters by LSE can be obtained in two stages:

1. First estimation of Weibull parameters: $\hat{\eta}_{k}$ and $\hat{\beta}_{k}$, for $k=1$ to $\mathrm{s}$. The distribution of failure times at the first step is fitted by a Weibull model and a first estimation of $\eta_{1}$ and $\beta_{1}$ is deduced.

For the other steps $k \geqslant 2$, the failure times $t_{i}$ cannot be fitted by a Weibull distribution at the beginning. They have first to be changed into equivalent times, as detailed in Section 2, with a shift $A_{k}$ so that the resulting equivalent times follow a Weibull distribution.

As it has already been noticed for the relationship (4):

for any $t_{i} \leqslant \tau_{1}$,

$$
t_{i, 1}=t_{i}
$$

and, for any $k \geqslant 2$ and $\tau_{k-1}<t_{i} \leqslant \tau_{k}$,

$$
t_{i, k}=t_{i}+A_{k}
$$

where the shift $A_{k}$ depends on the Weibull parameters at the stress levels of steps 1-k.

As the equivalent times $t_{i, k}$ represent times to failure for a two-censored test at the single stress $x_{k}$, they have to follow a Weibull distribution. Thus, the listplot $\left(\log t_{i, k}, \log \left[-\log \left(F_{i}^{\mathrm{e}}\right)\right]\right)$ defined for all the $t_{i}$ at step $k$, where $F_{i}^{\mathrm{e}}$ denotes the empirical frequency at the time $t_{i}$, has to be fitted by a Weibull straight line.

Thus, we suggest estimating the shift $A_{k}$ by $\hat{A}_{k}=\operatorname{ArgMax}_{A_{k}}\left(\rho_{k}\right)$ where

- $\rho_{k}$ denotes the correlation factor between the $x$ and $y$ coordinates of the previous listplot.

- ArgMax gives the value of $A_{k}$ for which $\rho_{k}$ is maximum.

Let us remember that:

- the empirical frequency is defined for each time to failure $t_{i}$, for $i$ equals 1 to the sample size $N$, by $F_{i}^{\mathrm{e}}=(i-0.3) /(N+0.4)$;

- the correlation factor between two variables $x$ and $y$ is defined by $\rho=\operatorname{cov}(x, y) / \sigma(x) \sigma(y)$, where cov denotes the covariance and $\sigma$ the standard deviation.

The optimization process used for the estimations of $A_{k}$ still has to be initialized but the starting value of the optimization process can be found very close to the solution thanks to the method detailed in Appendix A.

Then, the estimations $\hat{\eta}_{k}$ and $\hat{\beta}_{k}$ are the specific parameters of the Weibull probability plot defined for the equivalent times $t_{i, k}$ (at step $k$ ) for the estimated shift $\hat{A}_{k}$ obtained.

This first stage only estimates the Weibull parameters from times to failures at one single step, which may lead to a great inaccuracy. Thus, it will be worth improving these estimations by processing the times to failure altogether, especially for the parameters at the last step (step s) defined for the nominal level.

2. Improved estimations: $\hat{\eta}_{k}^{\prime}$ and $\hat{\beta}_{k}^{\prime}$, for $k=2$ to $s$. For given values of parameters, equivalent failure times $t_{i, k}$ corresponding to $t_{i} \leqslant \tau_{k-1}$ can be calculated by iterations on $k$ with (5) and

$$
t_{i, k}=\eta_{k}\left(\frac{t_{i, k-1}}{\eta_{k-1}}\right)^{\phi_{k}}
$$

where $\phi_{k}$ represents $\beta_{k-1} / \beta_{k}$. 


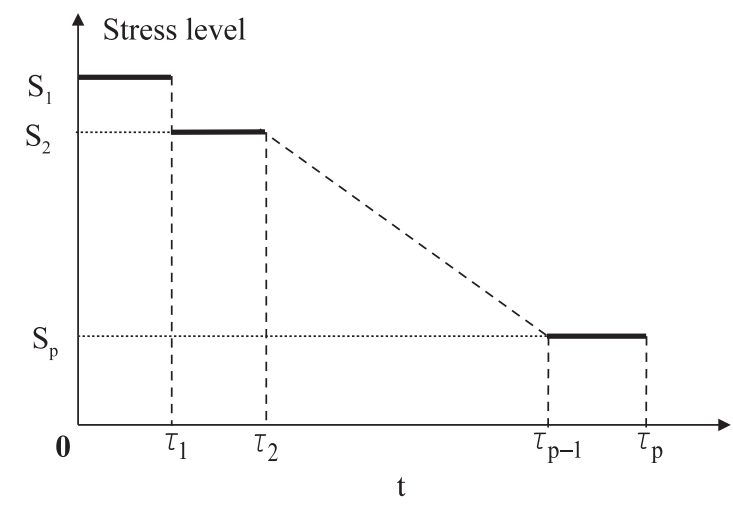

Fig. 3. Test profile.

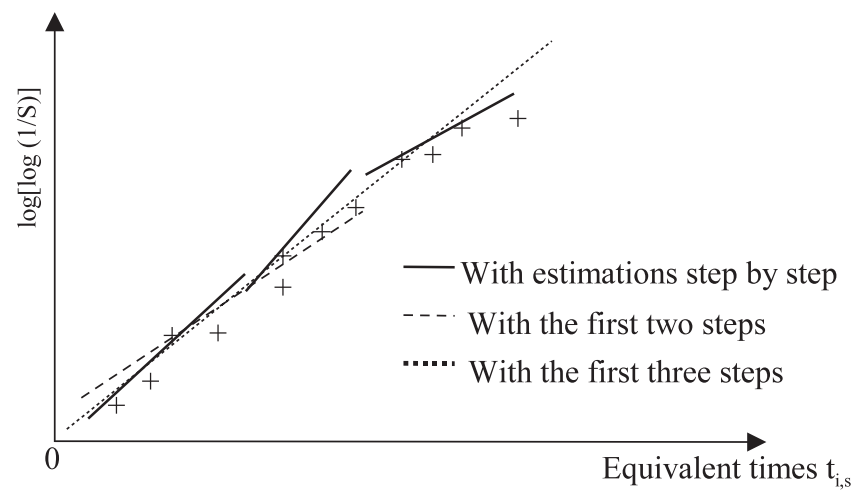

Fig. 4. Weibull probability plots.

Those corresponding to $t_{i}>\tau_{k-1}$ can be given by (6) with

$$
A_{k}=\eta_{k}\left(\frac{\tau_{k-1}}{\eta_{k-1}}\right)^{\phi_{k}}-\tau_{k-1}
$$

For any $k \geqslant 2$, an estimation of these parameters taking into account all the failure times until $\tau_{k}$ is given by

$$
\left(\hat{\eta}_{k-1}^{\prime}, \hat{\eta}_{k}^{\prime}, \hat{\phi}_{k}^{\prime}\right)=\underset{\eta_{k-1}, \eta_{k}, \phi_{k}}{\operatorname{ArgMax}}\left(\rho_{k}^{\prime}\right)
$$

where $\rho_{k}^{\prime}$ denotes the correlation factor between all the equivalent times $t_{i, k}$ defined for $t_{i} \leqslant \tau_{k}$ and the empirical frequencies $F_{i}^{e}$ in a Weibull scale.

The Weibull probability plot obtained for these parameter values gives the estimation $\hat{\beta}_{k}^{\prime}$ and, thus, the improved estimation of $\beta_{k-1}$ is given by

$$
\hat{\beta}_{k-1}^{\prime}=\hat{\phi}_{k}^{\prime} \cdot \hat{\beta}_{k}^{\prime} \text {. }
$$

When this estimation method is carried out until the step $s$, the parameters $\eta_{s}$ and $\beta_{s}$ will be estimated from all the times to failure of the whole test.

This method can be illustrated for a test with $s$ decreasing levels (Figs. 3 and 4).

Then, a bootstrap method can be used to improve the estimates and to obtain Cl (see Efron and Tibsharani, 1993). It consists of simulating a lot of step-stress ALT from a single one by re-sampling the failure times obtained. For each re-sampling, $N_{\mathrm{f}}$ new failure times are chosen with possible repetitions among the $N_{\mathrm{f}}$ obtained at the end of the test and the parameters of interest are estimated.

It was shown that the mean of the estimates obtained after each re-sampling represents a corrected estimate and that the standard deviation of the estimates obtained after each re-sampling represents the standard deviation of estimates for several actual tests. Any fractile may be estimated by the same way. Then, $\mathrm{CI}$ of parameter estimates can be defined. 
It can be noticed that the first estimations of Weibull parameters require to maximize a correlation factor with respect to one single parameter (the shift) with a starting value close to the final one. They can be improved by maximizing this correlation factor with respect to three parameters only $\left(\phi_{k}, \eta_{k}\right.$ and $\left.\eta_{k-1}\right)$, with starting values close to the final ones too.

Thus, the problems of initialization encountered with the MLE method will be solved much easier with this alternative method. Moreover, like the MLE method, this new method also enables one to estimate CI for parameter estimates.

To verify the efficiency of this new method, results of several test simulations with two or three steps will now be presented.

\section{Examples}

\subsection{Step-stress test simulations}

The graphical method proposed has been carried out on several tests simulations with $s=2,3$ and 4 steps. The tests can be simulated with given stress levels $x_{k}(k=1$ to $s)$ by generating random times to failure $t_{i}$ as follows:

- First of all, $N$ equivalent times $t_{i}^{\text {sim }}$ are generated from a Weibull distribution with given parameters $\eta_{s}$ and $\beta_{s}$ and sorted.

- The equivalent times $\tau_{k, s}$ to the end of steps $k$ are calculated with the relationships:

$$
\begin{cases}\tau_{1,1}=\tau_{1} \quad \text { and } \quad \tau_{k, k}=\tau_{k}+\eta_{k}\left(\frac{\tau_{k-1, k-1}}{\eta_{k-1}}\right)^{\frac{\beta_{k-1}}{\beta_{k}}} & \text { for } k=2 \text { to } s \\ \tau_{k, s}=\eta_{s}\left(\frac{\tau_{k, k}}{\eta_{k}}\right)^{\frac{\beta_{k}}{\beta_{s}}} & \text { for } k=1 \text { to } s-1\end{cases}
$$

- The simulated equivalent times $t_{i}^{\text {sim }}$ greater than $\tau_{s, s}$ are suppressed.

If $\tau_{s-1, s} \leqslant t_{i}^{\text {sim }}<\tau_{s, s}, t_{i}=t_{i}^{\text {sim }}-\tau_{s, s}+\tau_{s}$.

If $t_{i}^{\text {sim }}<\tau_{s-1, s}$, it is changed to $\eta_{s-1}\left(t_{i}^{\operatorname{sim}} / \eta_{s}\right)^{\beta_{s} / \beta_{s-1}}$.

All the simulated equivalent times to failure in the intervals $\left[\tau_{k-1, s}, \tau_{k, s}\right]$ from $k$ equals $s-1$ to 1 can be processed by this way, that is:

If $\tau_{k-1, s} \leqslant t_{i}^{\text {sim }}<\tau_{k, s}, t_{i}=t_{i}^{\text {sim }}-\tau_{k, s}+\tau_{k}$.

If $t_{i}^{\text {sim }}<\tau_{k-1, s}, t_{i}^{\text {sim }}$ is changed to $\eta_{k-1}\left(t_{i}^{\text {sim }} / \eta_{k}\right)^{\beta_{k} / \beta_{k-1}}$.

For the profile shown in Fig. 3, the generation of times to failure is illustrated in Fig. 5.

Remark. For this paper, the step times of the simulated tests have been calculated to obtain a significant number of failures at each step. In practice, Weibull parameters are unknown and the number of stress levels required may be unknown too. In this case, each step can be ended when a minimum number of failures are obtained. This kind of plan would have been more complicated to simulate whereas this paper only aims to prove the efficiency of the method to deal with a lot of steps. Anyway, it could be processed with the same method.

\subsection{Results of simulations with two steps}

A first simulation with two steps was carried out with the following parameters: $N=200, \eta_{1}=3.76, \beta_{1}=2.94, \eta_{2}=100$ and $\beta_{2}=1.5$.

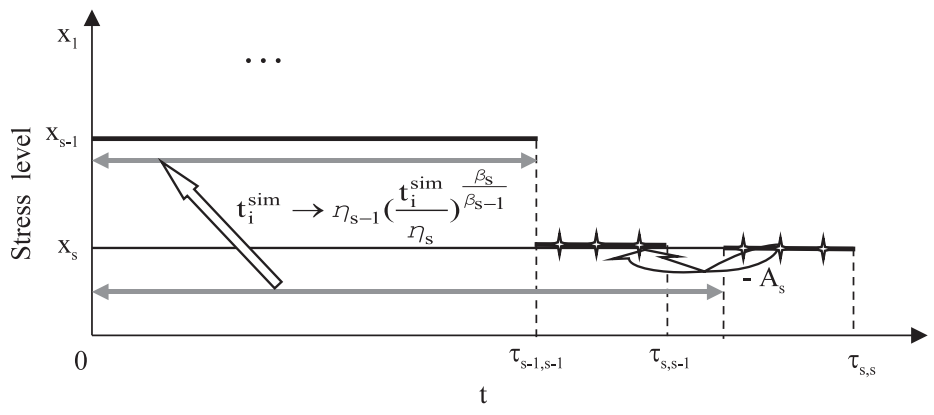

Fig. 5. Generation of times to failure in a step-stress test. 


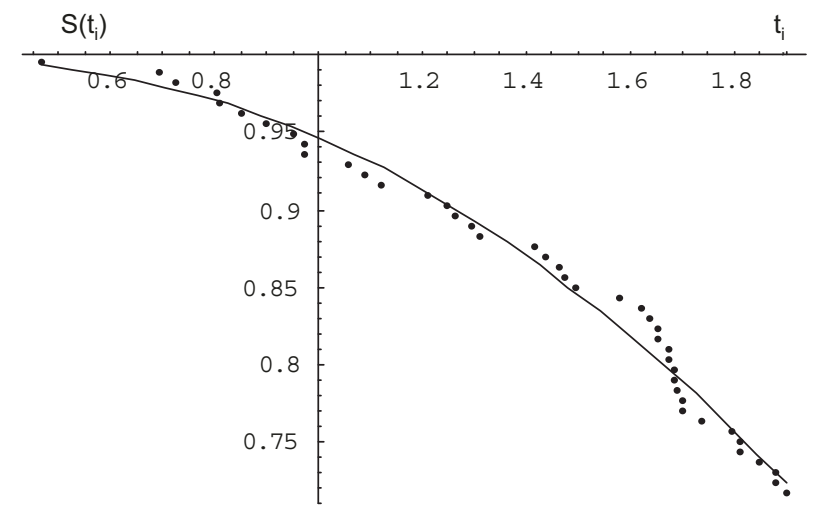

Fig. 6. Weibull plot for the first step.

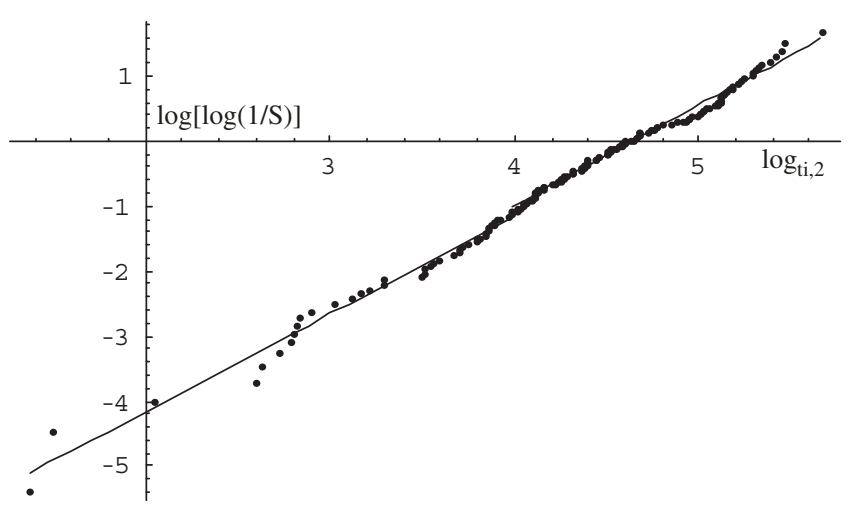

Fig. 7. Weibull plot for the two steps separately.

The step times have been defined so that 90 units fail at the first step, 90 others at the second step, and 20 failures are censored. Then simulations and a process of results have been carried out as follows:

- $N$ times to failure $t_{i}$ were generated.

- The times to failure of the first step were processed. A first estimation of $\eta_{1}$ and $\beta_{1}$ was obtained (see Fig. 6) by fitting a Weibull model to simulation results.

It led to the estimated parameters $\hat{\eta}_{1}=4.68$ and $\hat{\beta}_{1}=2.17$.

- The times to failure of the second step were processed. The correlation factor $\rho$ between the corresponding equivalent times $t_{i, 2}=t_{i}+A_{2}$ and the estimations of the cumulative distribution function (in a Weibull scale) was optimized with respect to $A_{2}$. It led to $\hat{A_{2}}=56.7$.

The resulting Weibull probability plot is shown in Fig. 7.

It led to $\hat{\eta}_{2}=119.58$ and $\hat{\beta}_{2}=1.51$.

It can be seen in Fig. 7 that the Weibull probability plot obtained for equivalent times at the nominal stress is slightly different from the first step to the second one.

- A second estimation of $\eta_{2}$ and $\beta_{2}$ was obtained by gathering the equivalent failure times of both steps. The correlation factor for this second list was optimized again and it led to $\hat{\eta}_{2}=99.04$ and $\hat{\beta}_{2}=1.30$.

The resulting Weibull probability plot is shown in Fig. 8.

Table 1 gives the results obtained by simulation of $n=100$ and 500 samples with a size $N=200$ and the mean square errors are indicated.

The accuracy and the robustness of the method can be pointed out because, for a test at a single stress level $x_{2}$ with the same sample size and test time, the MS error on $\eta_{2}$ and $\beta_{2}$ is about 100 times higher.

This accuracy can be improved thanks to the bootstrap method. For 1000 bootstrap replications among the results obtained from one simulation led to 1000 estimations of parameters. Their values are shown on the following histograms (Figs. 9 and 10). 


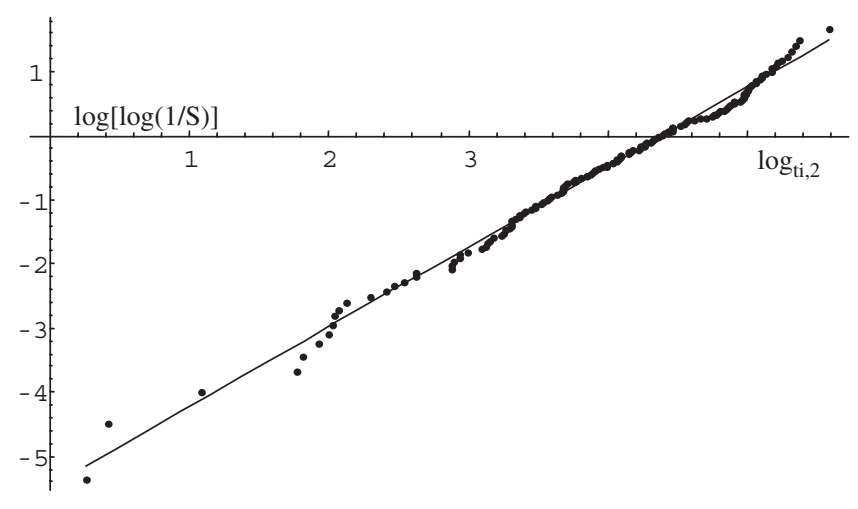

Fig. 8. Weibull plot showing the equivalent times of both steps.

Table 1

Estimation accuracy with two steps

\begin{tabular}{|c|c|c|c|c|c|}
\hline Parameter & True value & Mean $(n=100)$ & MS error & Mean $(n=500)$ & MS error \\
\hline$\eta_{2}$ & 96 & 99.43 & 0.06 & 99.08 & 0.06 \\
\hline$\beta_{2}$ & 1.43 & 1.51 & 0.06 & 1.51 & 0.06 \\
\hline$\eta_{1}$ & 3.76 & 3.80 & 0.05 & 3.80 & 0.05 \\
\hline$\beta_{1}$ & 2.94 & 2.91 & 0.04 & 2.89 & 0.03 \\
\hline
\end{tabular}

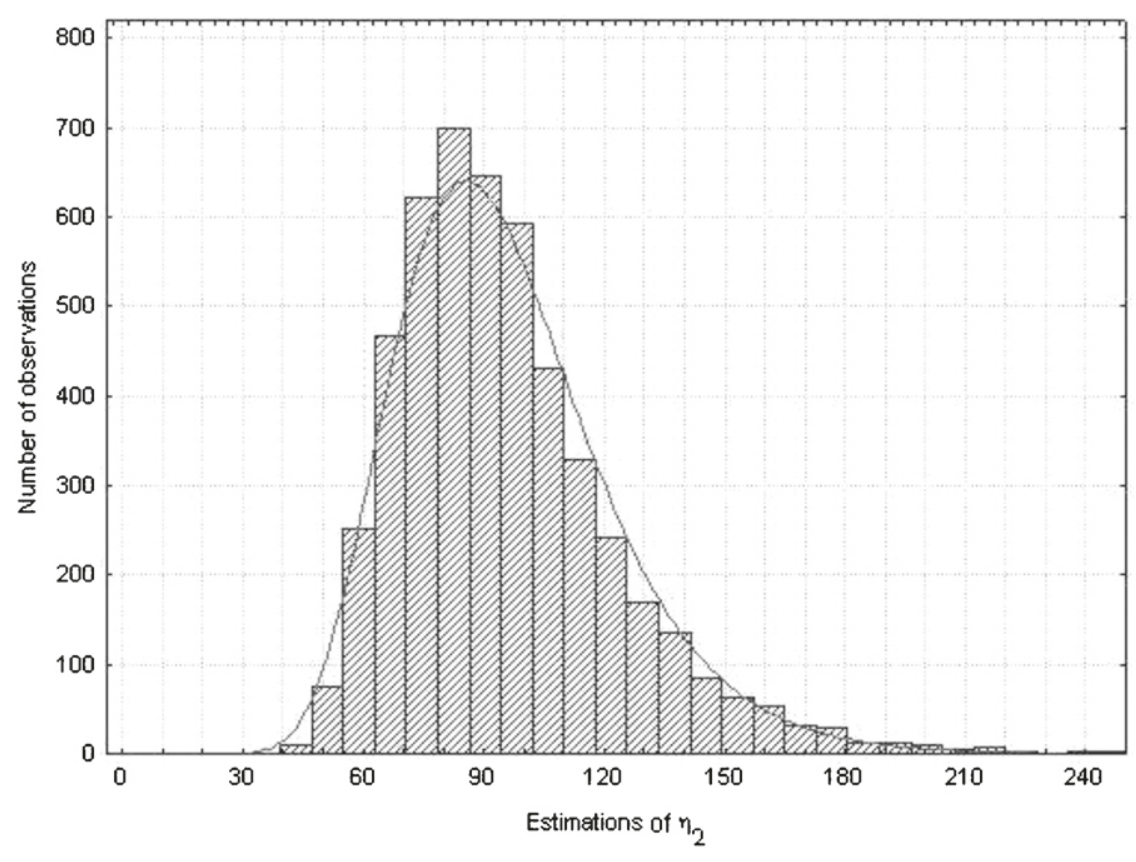

Fig. 9. Lognormal distribution for $\eta_{2}$.

In this case, as shown in these two figures, the distribution of estimators can be fitted by a lognormal distribution. The goodness of fit has been verified by a chi-square test at a $1 \%$ significance level.

Knowing the distribution of estimations, two-sided $\mathrm{Cl}$ at a confidence level (CL) $\alpha=90 \%$ have been defined. Results are shown in Table 2.

Not only are the corrected estimations always closer than the first ones to the true values but Cl obtained at a 90\% CL always bound the true values. 


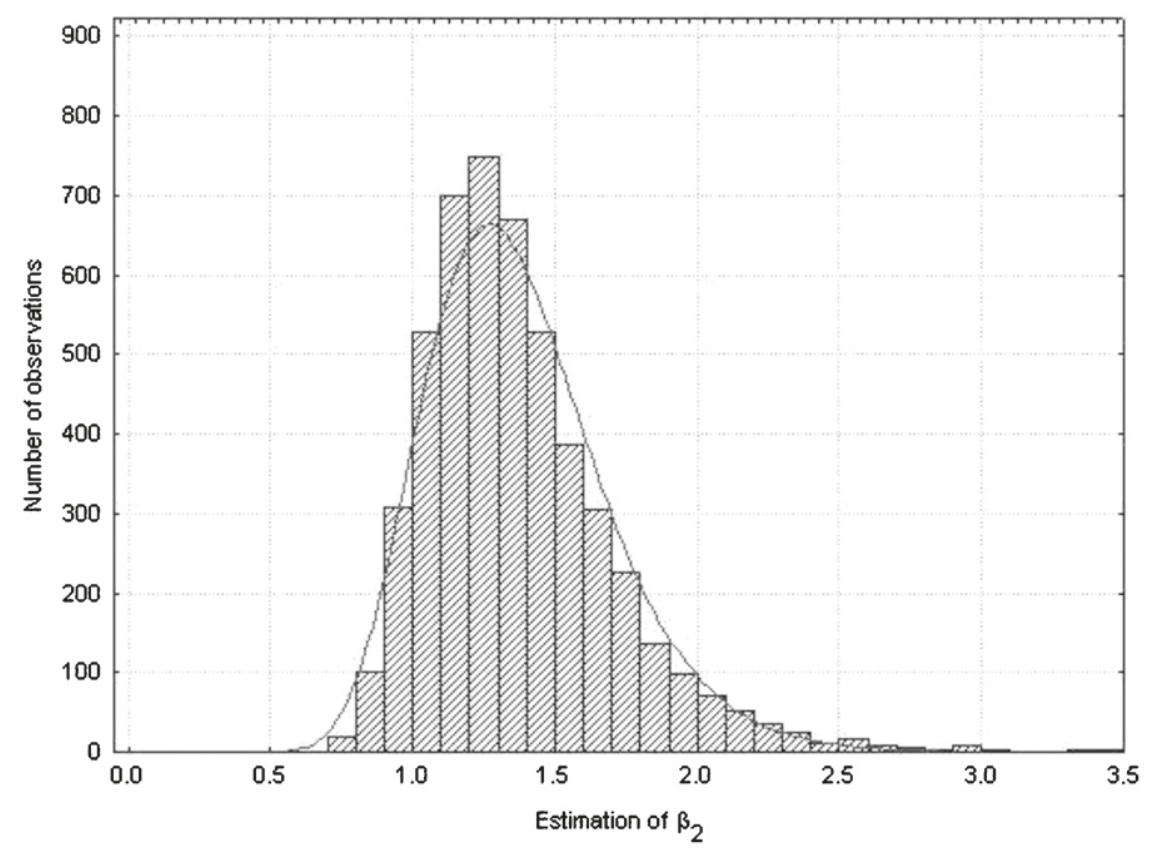

Fig. 10. Lognormal distribution for $\beta_{2}$.

Table 2

Corrected estimations and confidence intervals

\begin{tabular}{|c|c|c|c|c|}
\hline Parameter & $\eta_{2}$ & $\beta_{2}$ & $\eta_{1}$ & $\beta_{1}$ \\
\hline True value & 96 & 1.43 & 3.76 & 2.94 \\
\hline First estimation & 89 & 1.57 & 3.62 & 3.07 \\
\hline Corrected estimation & 96.2 & 1.38 & 3.76 & 2.96 \\
\hline $\mathrm{CI}(\mathrm{CL}=90 \%)$ & {$[94.8 ; 100.3]$} & {$[1.32 ; 1.48]$} & [3.69;3.89] & {$[2.90 ; 3.08]$} \\
\hline
\end{tabular}

\subsection{Results of simulations with three steps}

To evaluate the influence of the number of steps, a medium step was added at a stress level between the two previous ones. The Weibull parameters at this level are $\eta_{1}=8.3$ and $\beta_{1}=2.18$ and the test time is the same as that of the two-step test.

The first simulation led to $\hat{\eta}_{3}=113.42$ and $\hat{\beta}_{3}=1.56$; the Weibull plot obtained for all the equivalent times with steps processed separately is shown in Figs. 11 and 12 when they are processed all together.

Table 3 gives the results obtained by simulation of $n=100$ and 500 samples with a sample size $N=200$.

As a conclusion, the evolution of the survival function at the nominal stress level has been compared to the estimated survival functions obtained with mean estimated parameters for 100 and 500 simulations (Fig. 13).

\subsection{Application to a common test profile}

To demonstrate that this method enables one to use common test profiles, a test with four steps including two increases and one decrease in the stress level has been used. We have also used the bootstrap method to improve estimations and to show that, despite the numerous steps and the complicated profile, good accuracy can be obtained.

The simulation was carried out with the following parameter values and with a sample size $N=300$. The step times and the scale and shape parameters $\eta_{k}$ and $\beta_{k}$ (for $k=1-4$ ) chosen for the simulations are given in Table 4 .

The test profile is shown in Fig. 14.

This test profile can be simulated like in Section 4.1. The distribution of failure times obtained and the first estimations of the Weibull parameters at each stress level are given in Table 5.

Table 6 gives the second estimations of Weibull parameters obtained after gathering all the equivalent failure times without and with the bootstrap correction (for 5000 sample repetitions), the estimated coefficients of variation (CV) and two-sided CI at the level $90 \%$. 


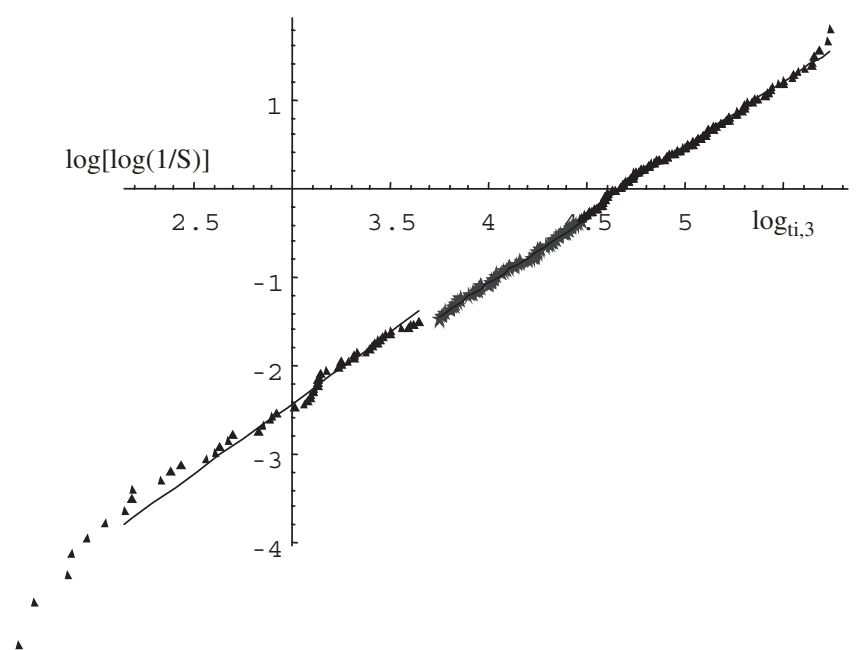

Fig. 11. Steps processed separately.

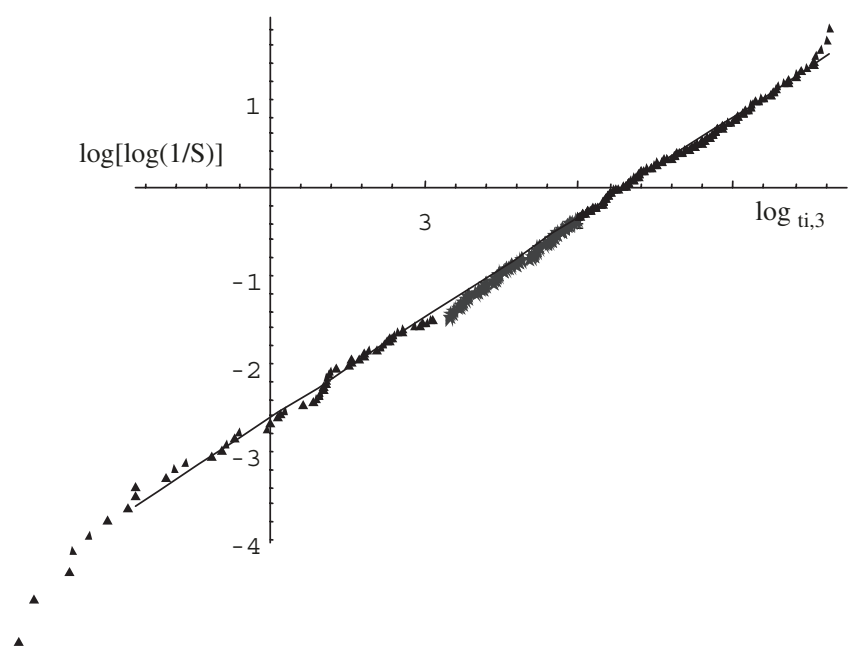

Fig. 12. Steps processed together.

Table 3

Estimation accuracy with three steps

\begin{tabular}{|c|c|c|c|c|c|}
\hline Parameter & True value & Mean for $n=100$ & MSE & Mean for $n=500$ & MSE \\
\hline$\eta_{3}$ & 96 & 77.32 & 0.12 & 103.28 & 0.10 \\
\hline$\beta_{3}$ & 1.43 & 1.58 & 0.08 & 1.53 & 0.09 \\
\hline$\eta_{2}$ & 48.3 & 53.29 & 0.07 & 43.7 & 0.06 \\
\hline$\beta_{2}$ & 2.18 & 2.54 & 0.06 & 1.92 & 0.04 \\
\hline$\eta_{1}$ & 3.76 & 3.91 & 0.05 & 3.62 & 0.04 \\
\hline$\beta_{1}$ & 2.94 & 2.70 & 0.05 & 3.04 & 0.05 \\
\hline
\end{tabular}

Except for the first step (with quite few failures), the CV is always lower than $5 \%$. Moreover, the true values always belong to the $\mathrm{CI}$ found. It means that one can rely on the information given by this estimation method even for complicated test profiles.

\section{Conclusion}

If one only knows a lifetime distribution model and if nothing is known about the acceleration model, it is still possible to estimate the reliability of a product at a nominal stress level with a step-stress accelerated test. This paper has shown that estimations may remain accurate, even for a lot of steps. 


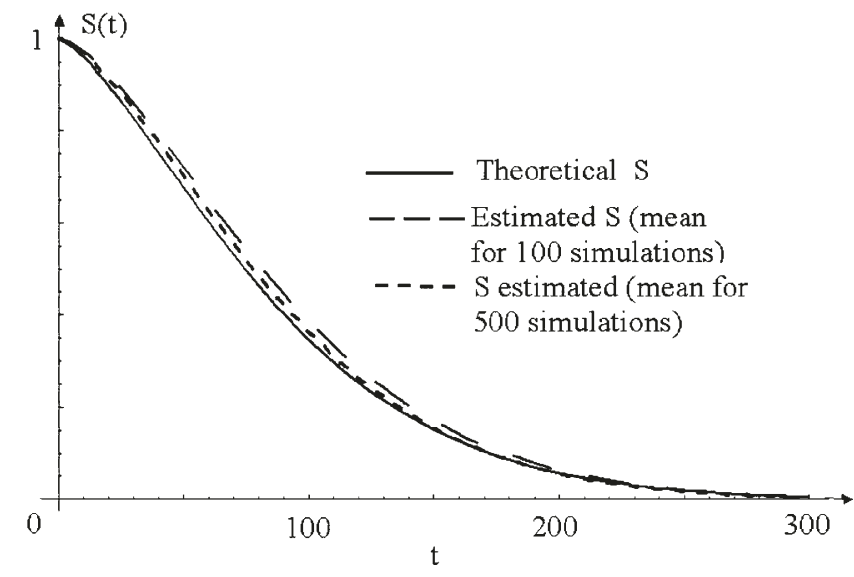

Fig. 13. Steps processed together.

Table 4

Parameters values for the simulation

\begin{tabular}{lcccc}
\hline Step number & 1 & 2 & 3 & 4 \\
\hline$\eta_{k}$ & 100 & 80 & 5 & 3 \\
$\beta_{k}$ & 1.5 & 2 & 3 & 150 \\
Step times & 30 & 20 & 2 & 1.2 \\
\hline
\end{tabular}

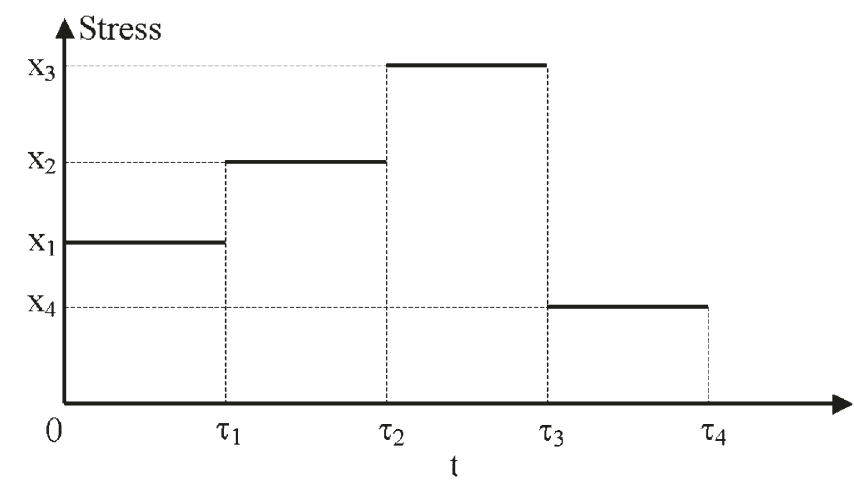

Fig. 14. Common test profile.

Table 5

First estimation of parameters

\begin{tabular}{lllll}
\hline Step number & 1 & 2 & 3 & 4 \\
\hline Number of failures & 38 & 139 & 67 & 44 \\
Estimations & $(80.2 ; 2.0)$ & $(4.7 ; 2.6)$ & $(200 ; 2.5)$ & $(5 ; 3)$ \\
True values & $(100 ; 1.5)$ & $(80 ; 2)$ & $(150 ; 1.2)$ \\
\hline
\end{tabular}

With previous MLE methods, processing all the failures led to estimate a lot of parameters and thus to increase the calculation time when the initialization was far from the true values. The LSE method presented in this paper still requires an initialization for each step but, as the starting values of parameters can be found close to the true ones, this method can lead to better precisions within a shorter calculation time.

As this precision can be controlled with both methods, it is always possible to know which of the LSE or the MLE method leads to the best accuracy. Even if the MLE is better, it is worth using the LSE method at the beginning to initialize the MLE method. Thus, the LSE presented in this paper always improves the estimation process used after an ALT. 
Table 6

Estimations of parameters $\eta_{k}$ and $\beta_{k}$

\begin{tabular}{llll}
\hline Step number & 1 & 2 & 3 \\
\hline True values & $(100 ; 1.5)$ & $(80 ; 2)$ & $(5 ; 3)$ \\
Without bootstrap & $(80.2 ; 2.0)$ & $(113 ; 0.93)$ & $(4.7 ; 2.6)$ \\
With bootstrap & $(93.8 ; 1.39)$ & $(82.3 ; 2.07)$ & $(5.00 ; 3.01)$ \\
Estimated CV & $(6.8 \% ; 7.5 \%)$ & $(2.9 \% ; 2.3 \%)$ & $(150 ; 1.2)$ \\
CI $(C L=90 \%)$ & {$[88.2 ; 102.3]$ and $[1.49 ; 1.63]$} & {$[78 ; 87]$ and $[1.97 ; 2.24]$} & {$[4.98 ; 5.06]$ and $[2.99 ; 3.02]$}
\end{tabular}

\section{Appendix A. Starting value for the estimation of the shift $A_{k}$}

We have shown that shifted failure times $t_{i}+A_{k}$ at a given step $k$ may follow a Weibull distribution, but the shift $A_{k}$ has to be correctly estimated.

Let us denote by $t_{\min }^{(k)}$ and $t_{\max }^{(k)}$ the first and last failure times at step $k$, and $Y=\log [\log (1 / S)]$.

The time $t_{m}$ for which $Y\left(t_{m}\right)=Y\left(t_{\min }^{(k)}\right)+Y\left(t_{\max }^{(k)}\right) / 2$ can be estimated by a linear interpolation of $Y$. The corresponding relationship leads to

$$
\beta_{k}\left[\log \left(t_{m}-A_{k}\right)-\log \left(\eta_{k}\right)\right]=\frac{\beta_{k}}{2}\left[\log \left(t_{\min }^{(k)}-A_{k}\right)\left(t_{\max }^{(k)}-A_{k}\right)-2 \log \left(\eta_{k}\right)\right]
$$

Thus

$$
A_{k}=\frac{\left(t_{m}\right)^{2}-t_{\min }^{(k)} \cdot t_{\max }^{(k)}}{2 t_{m}-t_{\min }^{(k)}-t_{\max }^{(k)}}
$$

It can be noticed that very few failure times have been taken into account (the first and last ones and the closest to $t_{m}$ ). That is why it has been pointed out that the estimations obtained by this way only enable one to initialize the process detailed in this paper.

\section{References}

Bagdonavicius, V., Nikulin, M., 1995. Semi-parametrics Models in Accelerated Life Testing. Queen's Paper in Pure and Applied Mathematics, vol. 98. Queen's University, Kingston, Canada.

Bagdonavicius, V., Nikulin, M., 2001. Mathematical Models in the Theory of Accelerated Experiments. Vol: Mathematics and the 21st Century. World Scientific, Cairo University, Egypt, pp. 271-273.

Bagdonavicius, V., Cheminade, O., Nikulin, M., 2004. Statistical planning and inference in accelerated life testing using the CHSS model. J. Statist. Plann. Inference $126(2), 535-551$.

Efron, B., Tibsharani, R., 1993. An Introduction to Bootstrap. Chapman \& Hall, New York.

Mc Linn, J.A., 1998. Ways to improve the analysis of multi-level accelerated life testing. Qual. Reliab. Eng. Internat. 14, 393-401.

Nelson, W., 1990. Accelerated Testing: Statistical Models, Test Plans and Data Analysis. Wiley Series in Probability and Mathematical Statistics. Wiley, New York. Pham, H., 2003. Handbook of Reliability Engineering. Springer, Berlin.

Sedyakin, N.M., 1966. On one physical principle in reliability theory. Tech. Cybernet. 3, 80-87.

Tebbi, O., Guerin, F., Dumon, B., 2005. Standard accelerated life testing model applied to mechanical components. Internat. J. IEST 48 (1), $103-113$. 